\title{
DESIGN AND ANALYSIS OF MECHANISM TO APPLY PERIODIC DYNAMIC LOAD ON PVDF
}

\author{
Vikram (Corresponding Author) ${ }^{1} \&$ Deepak Chhabra ${ }^{2}$
}

\begin{abstract}
This paper presents the design and experimental verification of a mechanism which provides periodic dynamic load as and when required. Single piezoelectric full wave rectifier circuit, with three different boundary conditions, is analyzed by means of periodic dynamic load which is altered in each observation. The supports provided are simply support, circular support and fixed support. Circular support gives least variation in output voltage \& it also requires least no. of strokes (around 20). It has been observed that maximum voltage reading is traced by simply supported boundary condition. It is also observed that maximum force reading is traced by fixed support. The major aim of this paper is to give a picture of the best possible boundary condition for required features like output voltage, dynamic load etc.

Keywords: Piezoelectric Patch, single piezoelectric full wave bridge rectifier circuit, PVDF, force sensor, periodic dynamic load, power source, P.C.B.
\end{abstract}

\section{INTRODUCTION}

A dynamic load can be considered as live load acting on a structure or any object. Dynamic load on an object can be applied by various ways, like harmonic, impact, cyclic etc. Here we need to apply loading with varying magnitude in each observation. The value of load should not fluctuate within the individual observation. The all above stated conditions are fulfilled by designing a mechanism which apply periodic dynamic load. The mechanism is designed according to the requirement, considering into mind. Cyclic dynamic load with varying magnitude in each observation is applied on piezoelectric patch made of PVDF. This PVDF Piezo patch is employed with three different boundary conditions, namely simply supported, circular supported and fixed supported. Dynamic loading on PVDF Piezo patch is recorded by a force sensor. This force sensor read out the value of applied load in its rated unit. This unit is then calibrated with some suitable scale. It is seen that when mechanical type of action like loading is applied on Piezo patch, electricity is produced. The output voltage that is produced with the effect of mechanical loading is measured by connecting a Multimeter to the circuit. Here the circuit used is piezoelectric full wave bridge rectifier circuit and the details about circuit are given below in equipment section. Deepak Chhabra and Ankit Singroha (2017) uses diverse piezoelectric circuits to generate electricity and then employed output variables to run the miscellaneous devices like LED bulb, rechargeable cell or in storage process. Deepak Chhabra and Ashwani Kumar et al (2014) designed the EF model of cantilever beam with different cross sections like rectangular, triangular and rhomboids. They make use of ANSYS software for design purpose. Deepak Chhabra and Anmol Budhwar (2016) links between various factors for energy harvesting using single and double Piezo patches connected in different circuits. They perform experiments under hydraulic dynamism. Deepak Chhabra and Pooja Rani (2016) works on a model that provide a pressurized water jet on Piezo patch made up of PVDF. They shows the relation between energy extracted with various parameters such as space between Piezo patch and fluid nozzle, no. of nozzles and angle through which the water jet impinges on PVDF. Xu Wang and Liwei Lin (2013) made use of single load resist with 4 types of circuits for single degree of freedom Piezo energy harvester. Evgeniy L. Eremin and Evgeniy A. Shelenok (2013) simulated the tricky situations in electrodynamic shaker control and then amalgamate the shaker with different circuits which are based on hyper stability principle.

\footnotetext{
${ }^{1}$ Department of Mechanical Engineering, University Institute of Engineering \& Technology, Maharshi Dayanand University, Rohtak-124001 Haryana INDIA

${ }^{2}$ Department of Mechanical Engineering, University Institute of Engineering \& Technology, Maharshi Dayanand University, Rohtak-124001 Haryana INDIA
} 


\section{Main Parts and Construction of mechanism which Apply Periodic Dynamic Load on PVDF}

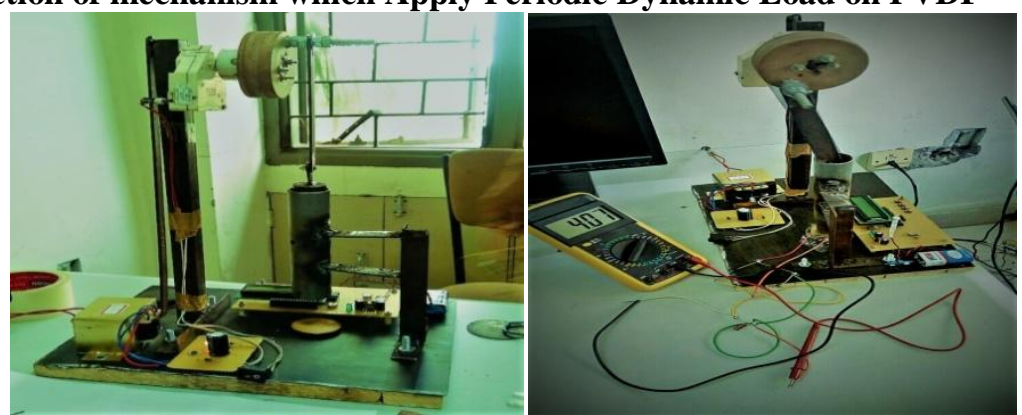

Fig: 1 Mechanism To Apply Periodic Dynamic Load On PVDF

$>$ Force sensors

$>$ Printed circuit board (P.C.B.)

$>$ Power supply

\subsection{Force Sensors}

Force sensors are highly durable that these can be used in various adverse conditions. These are used to measure force between 2 flat surfaces. In an electrical circuit, the Flexi force sensor acts as a force sensing resistor. When the force is eradicate from force sensors, resistance will be increasing \& vice versa. The device named Multimeter is used to measure the value of resistance. The dynamic load is applied on the stated surface under consideration. General applications in detection \& measurement with force sensors:

- Comparative variation in applied load or force

- Change of load w.r.t. time

- Force threshold \& generate proper exploit

- Contact \& touch

The construction features of Force sensor carry the advantages as given below:

- Fineness $\left(203 \times 10^{-3} \mathrm{~mm}\right)$

- Improved straightness

- To check the quantity of higher load

- Steady output w.r.t. Applied load vicinity on sensor

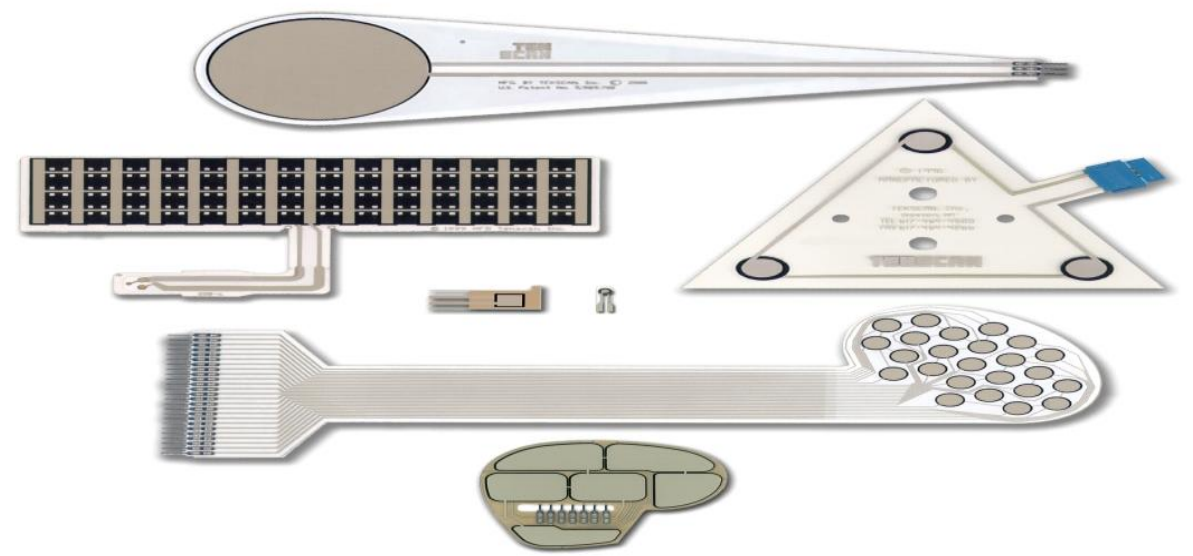

\subsection{Printed Circuit Board}

Fig: 2. Constructional Features Of Force Sensor

Printed Circuit Board are employed for shelter apparatus for making a circuit for firmness, ease of servicing \& in the case of internally connection. So P.C.B. can be defined as: This is a Bakelite sheet in which one side is employed by copper patterns with holes \& from another side, leads of electronic apparatus are inserted in the suitable holes \& soldered. By this way electronic circuit is prepared by joining the leads of electronic apparatus. Copper wrapping is done on the boards by thrashing slim copper foil on the boards during remedial. The copper wrapped thickness on the board is about $2 \mathrm{~mm} \&$ weights about 30 gm per square foot.

Boards are of two types of material

- (1) Phenolic paper based material

- (2) Glass epoxy material.

Both materials are available as thin sheets with copper wrapping. Printed circuit boards have a copper wrapping on one or may be on both sides. In both type of boards, pasting thin copper foil on the board does this. Boards are prepared in sizes of 1 to 5 meter wide \& up to 2 meters long. The board's thickness is 1.4 to $1.8 \mathrm{~mm}$. The copper thickness on the boards is about $0.2 \mathrm{~mm}$. 
P.C.B. requires following apparatus:-

- Copper(Cu) Wrapped Sheet

- Paint or Nail Polish

- $\mathrm{FeCl}$

- Plastic sheet

- Household Water etc.

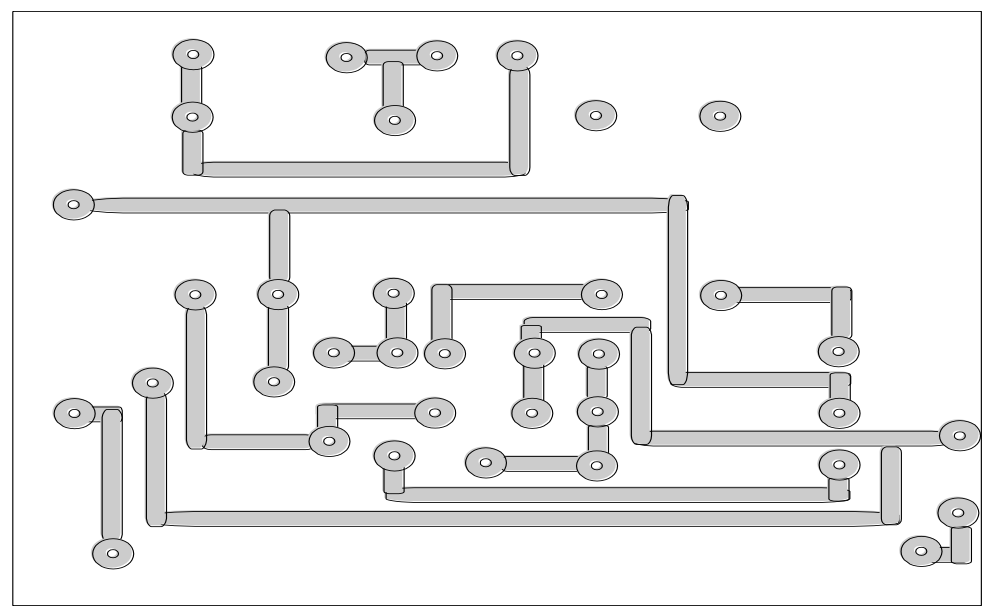

Fig: 3. Design Of Printed Circuit Board (P.C.B.)

There are some preliminary considerations, while making the P.C.B. drawing, such as width of lines or holes as per the apparatus. Then the P.C.B. sketch is drawn like tracks, rows, square as per the circuit diagram by using enamel paint, plain nail polish or any other acid resistant fluid. Arid the localized face in open air \& when it is absolutely dried, the noticeable holes in P.C.B. can be pierced with the help of $1 \mathrm{~mm}$ drill bit. In case of lack of lines due to spillage of paint, these may be detached by altercating with the help of a cutting edge or a knife by considering the point that the paint has been dried. The paint on the pattern can be eradicate over P.C.B. \& may be swabbed \& dried. Place a coat of shiny surface to retain the charm. Finally the P.C.B. is prepared for work.

The process of making a Printed Circuit board for any application has the following steps:

- Primarily layout was prepared.

- The prepared layout was transferred on the photographically M the copper.

- By a chemical process etching, the excessive copper was removed.

- Holes were drilled for mounting of components.

\section{Reactions:}

$\mathrm{FeCl}_{3}+\mathrm{Cu}------\mathrm{CuCl}_{3}+\mathrm{Fe}$

$\mathrm{FeCl}_{3}+3 \mathrm{H} 2 \mathrm{O}------\mathrm{Fe}(\mathrm{OH})_{3}+3 \mathrm{HCL}$

Precautions:

- Put in $\mathrm{FeCl}_{3}$ cautiously without any splashing because it may be unpleasant to the skin \& will mark the clothes.

- Place the copper side up to the printed circuit board in the solution.

- Don't inhale the vapour solution.

- Blend the solution by giving forced up \& down motion to the plate repeatedly \& put the solution on it.

- To ignore boiling, intermittently the solution is being warmed. After a while the unshared parts alter their clolor which are continued to etch. Progressively the base material will turn out to be visible. Etching for more than 2 minutes is done to obtain a neat pattern.

- Don't dispose of the remaining $\mathrm{FeCl}_{3}$ solution because it can be reused.

\subsection{Power Supply \& Requirement}

A 'power supply' is a primary requirement in case of giving energy or to deliver energy in some form. The batteries of solidstate electronic equipment can be removed by a battery eliminator \& thus the equipment can be operated by 230 volt A.C. mains as an alternative of the batteries or dry cells. These days the application of power supply or battery eliminator has become very much popular in the field of household appliances like transistors, cassette player, tape recorder, record player etc. 
In India, accessibility of electrical energy is in the shape of alternating voltage to residences \& industries. The electrical supply possess a voltage of $220 \mathrm{~V}$ for the frequency of $50 \mathrm{~Hz}$ whereas in the USA, the voltage of $110 \mathrm{~V}$ for the frequency of $60 \mathrm{~Hz}$. A DC voltage is needed for the satisfactory operation of the majority of the devices in electronic equipment. For illustration, a DC supply required by a transistor radio for its operation. Typically this is supplied by dry cells. Other than that, in place of dry cells, we can make use of a power supply. AC voltage is converted into DC voltage by the use of power source that eliminates the requirement of dry cells. At the present time, almost all-electronic apparatus comprises a circuit that changes $\mathrm{AC}$ voltage of mains supply into DC voltage and this is all done by power source. Usually a power transformer is applied at the entrance of the power supply \& a diode circuit called rectifier follow this. The output of the rectifier goes to a smoothing filter followed by a voltage regulator circuit. The important part of the power supply is rectifier circuit like heart for a human body. Rectification is the process of interpretation of an alternating current or voltage into a specified one way. Rectifier is used as the component for rectification. During the +ve half cycles only, the rectifier allows current to flow by eliminating the -ve half cycles or fluctuation of the applied AC voltage. Therefore effervescent DC is obtained. Additional filter circuits are employed to obtain smooth DC power.

A diode can be used as a rectifier. Even there are several types of diode but semiconductor diodes are very commonly used as rectifiers. Electron emitter and electron collector i.e. cathode and anode respectively, consisted by a solid-state device (a semiconductor diode). The unilateral conduction necessary for rectification is provided by the semiconductor diode because electrons become unidirectional flowing from emitter to collector. The two most commonly semiconductor diodes used are copper oxide \& selenium rectifier out of available diodes.

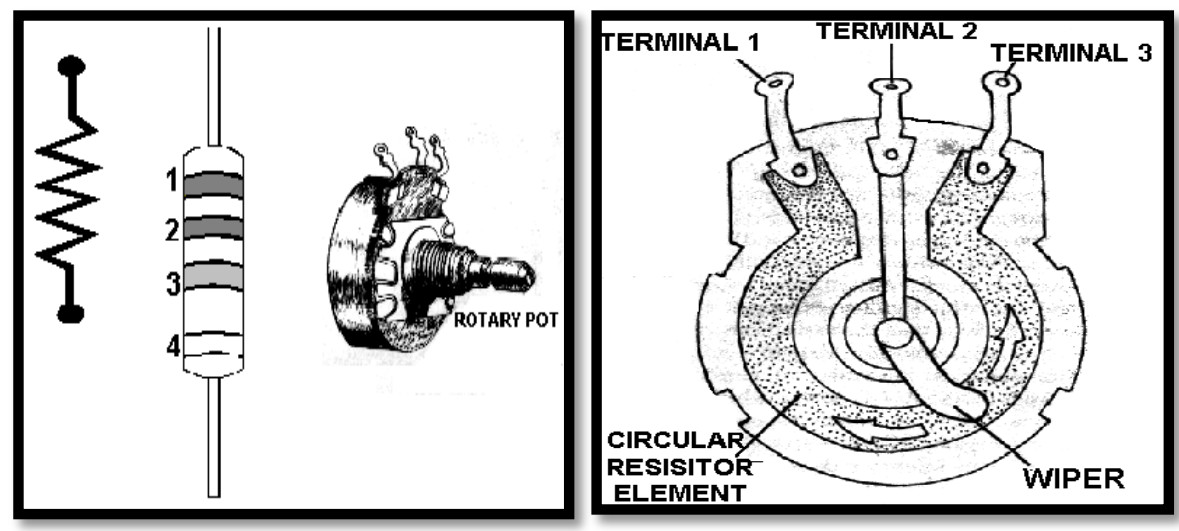

Fig: 4. Diode Rectifier

The $1^{\text {st }}$ and $2^{\text {nd }}$ digit are given by the $1^{\text {st }}$ and $2^{\text {nd }}$ rings respectively. The number of zeroes to be placed after the digits indicated by the $3^{\text {rd }}$ ring. Tolerance limit is given by the $4^{\text {th }}$ ring. There are the dial types of resistance boxes in alternating resistors. There is a button along with a metal indicator. This button forced down over brass pieces situated along a circle with some gap between each of them. Different valued resistance coils are coupled $b / w$ the space. The indicator moves over the brass pieces as the button rotates. If the space is skipped for once, then its resistance is integrated in the circuit. If the space is skipped for twice, then the resistance of both will be integrated in the circuit. Same phenomenon followed for next skipped spaces. The dial type of resistance box consists of several dials depending upon the range that it will be covering. When there is a need to read the value up to 10,000 by a resistance box, 3dials having 10 spaces are used i.e. there are 10 resistance coils every one having resistance 10. The $3^{\text {rd }}$ dial will be having 10resistances each of 100 . Because of the fact that the contact resistance of dial type of resistance boxes is small \& steady, thus the dial type of resistance boxes are more preferred.

\section{EQUIPMENT AND MATERIALS FOR EXPERIMENT}

\subsection{Piezoelectric Patch}

The piezoelectric patch is made up of thin coating PVDF (Poly Vinylidene Fluoride). This patch is rested between two layers of copper and silver electrodes. The general patterns of piezoelectric patch are:

Table: 1 . General pattern of piezoelectric patches

\begin{tabular}{|c|l|c|}
\hline $\mathbf{1 .}$ & Thickness of the circular patch & $0.85 \mathrm{~mm}$ \\
\hline $\mathbf{2 .}$ & Dia. of the circular patch & $40 \mathrm{~mm}$ \\
\hline $\mathbf{3 .}$ & Thickness of the copper electrode & $0.25 \mathrm{~mm}$ \\
\hline $\mathbf{4 .}$ & Thickness of the silver electrode & $0.13 \mathrm{~mm}$ \\
\hline
\end{tabular}




\begin{tabular}{|c|l|c|}
\hline $\mathbf{5 .}$ & Thickness of the PVDF layer & $0.55 \mathrm{~mm}$ \\
\hline
\end{tabular}

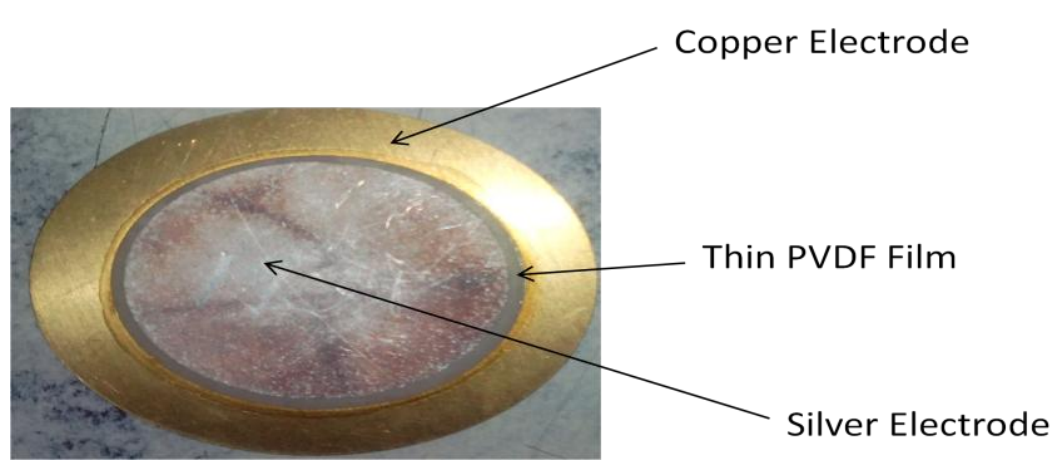

Fig: 5. Piezoelectric Patch

\subsection{Single Piezoelectric Full Wave Rectifier Circuit}

Single piezoelectric full wave rectifier circuit is usually applied to alter AC output into DC output voltage. Four diodes are consisted by this rectifying circuit. The rectifying phenomenon for output voltage is needed because a smooth and constant supply is required. With the help of smoothing capacitor, Piezo patch is connected to full wave rectifier circuit. When the periodic dynamic load is applied on the Piezo patch, multimeter measures the output voltage. The output voltage measured for different boundary conditions is discussed under in the form of table and suitable graph.

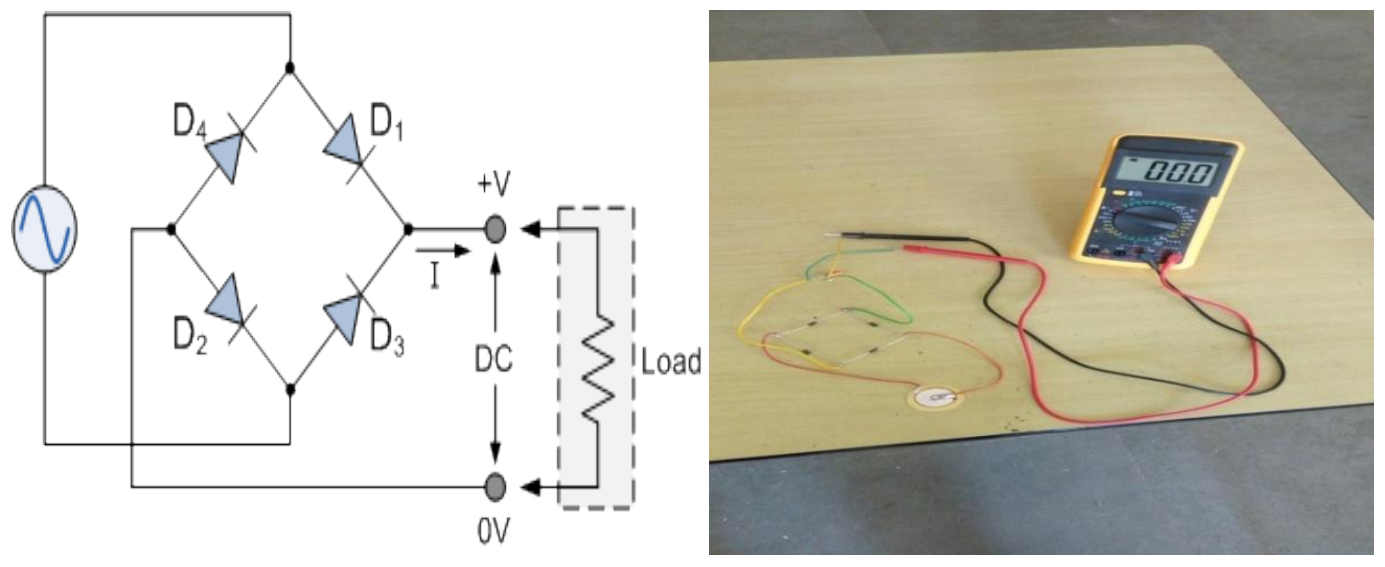

Fig: 6. Block Diag. Of Single Piezoelectric Full Wave Rectifier Circuit

\section{RESULT \& DISCUSSION}

To relatively assess the performance of Piezo patch with different boundary conditions against the fluctuating dynamic load, experiments were conducted. For these experiments single piezoelectric full wave rectifier circuit is employed. Three set of experiments were performed with given boundary condition.

\section{* General parameters considered for experiment on different boundary conditions}

○ Time for one Stroke $-2.2 \mathrm{sec}$.

$\circ$ No. Revolutions per minute $-27.27 \mathrm{rpm}$

$\circ$ Angular velocity $-2.8542 \mathrm{rad} / \mathrm{sec}$

$\circ$ Perimeter of wheel $-23 \mathrm{~cm}$

○ Radius of wheel $-3.7 \mathrm{~cm}$

$\circ$ Linear Velocity $-1.056 \mathrm{~cm} / \mathrm{sec}$ or $0.1056 \mathrm{~m} / \mathrm{s}$

\subsection{Simply Supported Boundary Condition}

First work is on simply supported boundary condition, which illustrate that while the intensity of load is increasing, the output voltage will be decreasing. This will shows a significant variation in no. of strokes which are ranging from 42 to $57 \&$ time taken by one observation varies from 93 seconds to 126 seconds. Load reading recorded by force sensor is ranging from 49 to 54. 

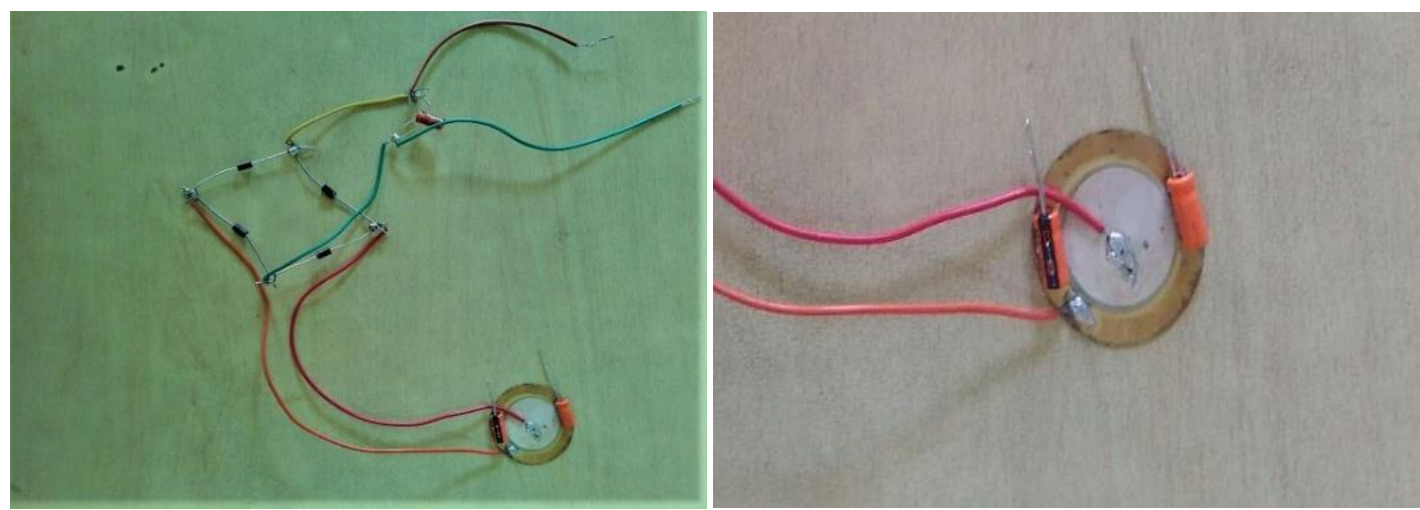

Fig: 6. Single Piezoelectric Full Wave Rectifier Circuit With Simply Supported Boundary Condition

Table: 2. Boundary condition: Simply Supported

\begin{tabular}{|l|l|l|l|}
\hline Sr. No. & Output voltage & Load Reading & No. of Strokes \\
\hline 1. & 13.70 & 49 & 42 \\
\hline 2. & 10.10 & 52 & 57 \\
\hline 3. & 9.20 & 54 & 52 \\
\hline
\end{tabular}

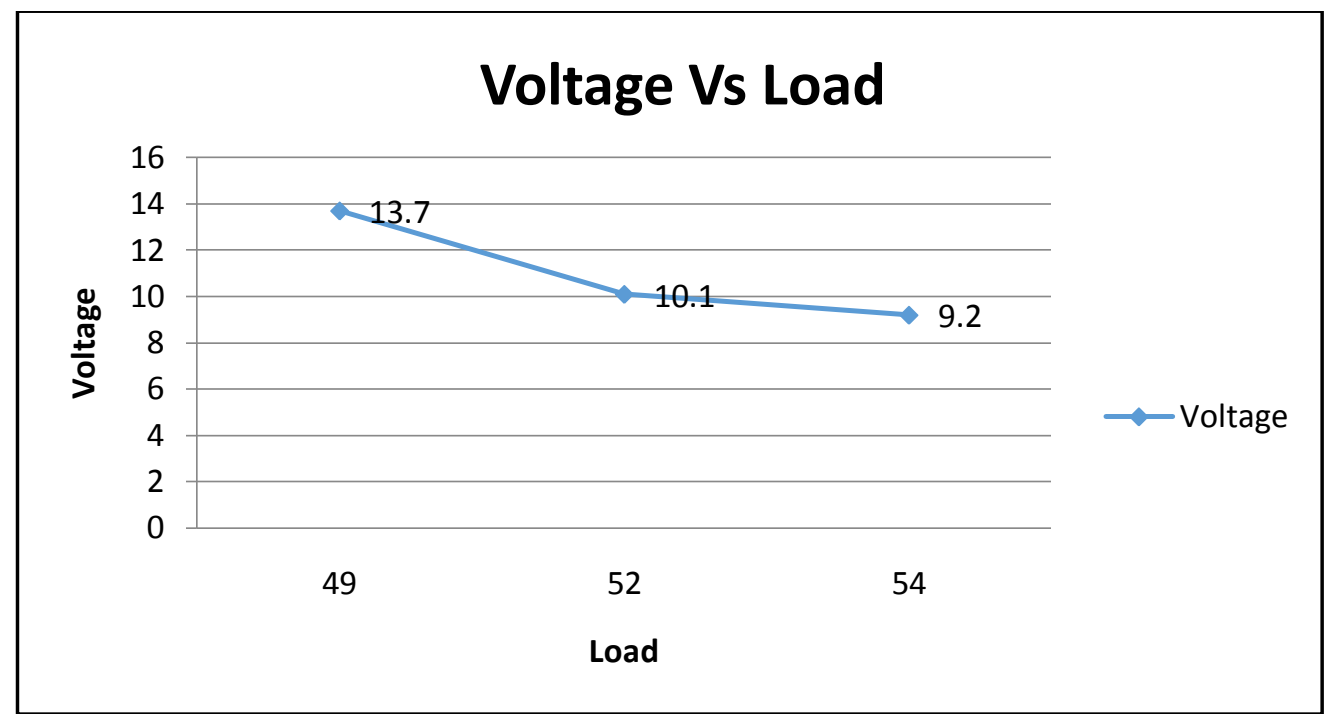

Fig: 7. Variation Of Voltage With Respect To Load Applied For Simply Supported Boundary Condition

\subsection{Circular Supported Boundary Condition}

Secondly circular supported Piezo patch is experimented that shows an increased reading in output voltage along with increased load. This phenomenon differs from simply supported condition in where opposite feature has been observed. The no. of strokes is almost same, around 20. Load reading fluctuates from 33 to 46 . Output voltage reading recorded by multimeter varies from 5.2 volt to 6.1 volt for the load reading 33 and 46 respectively.
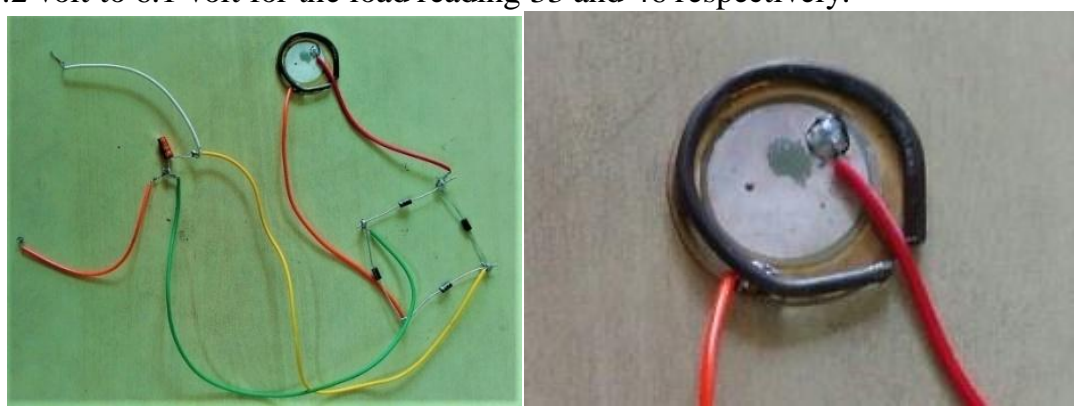

Fig: 8. Single Piezoelectric Full Wave Rectifier Circuit With Circular Supported Boundary Condition 
Table: 3. Boundary Condition; Circular Supported

\begin{tabular}{|l|l|l|l|}
\hline Sr. No. & Output voltage & Load Reading & No. of Strokes \\
\hline 1. & 5.20 & 33 & 20 \\
\hline 2. & 5.90 & 42 & 20 \\
\hline 3. & 6.10 & 46 & 19 \\
\hline
\end{tabular}

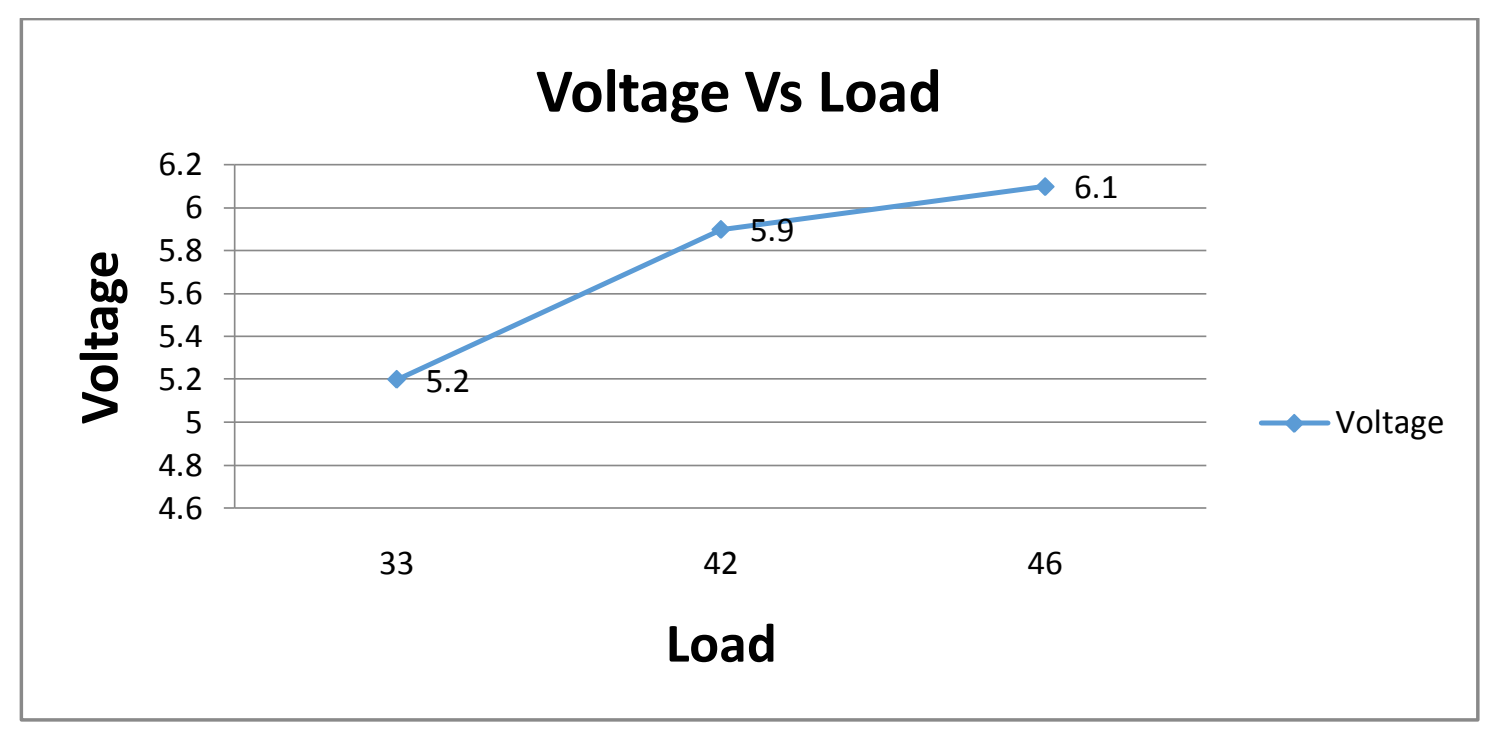

Fig: 9. Variation Of Voltage W.R.T. Load Applied For Circular Supported Boundary Condition

\subsection{Fixed Supported Boundary Condition}

Finally fixed supported boundary conditioned Piezo patch (single piezoelectric full wave rectifier circuit) is experimented. This condition shows a decrease in output voltage as the load value increasing and after some increment in load value the voltage reading on multimeter will become steady. So we can say that there is a reversing phenomenon. Here it is noted that load reading for different observations varies from 50 to 57 and at the same time output voltage recorded by multimeter is in the order of 7.5 volt to 3.85 volt respectively, which defines itself a reversing phenomenon. Total no. of strokes required for a stable reading varies from 35 to 43 . So by using time taken in a single stroke, we can calculate that the time taken by each observation varies from $77 \mathrm{sec}$ to $95 \mathrm{sec}$.
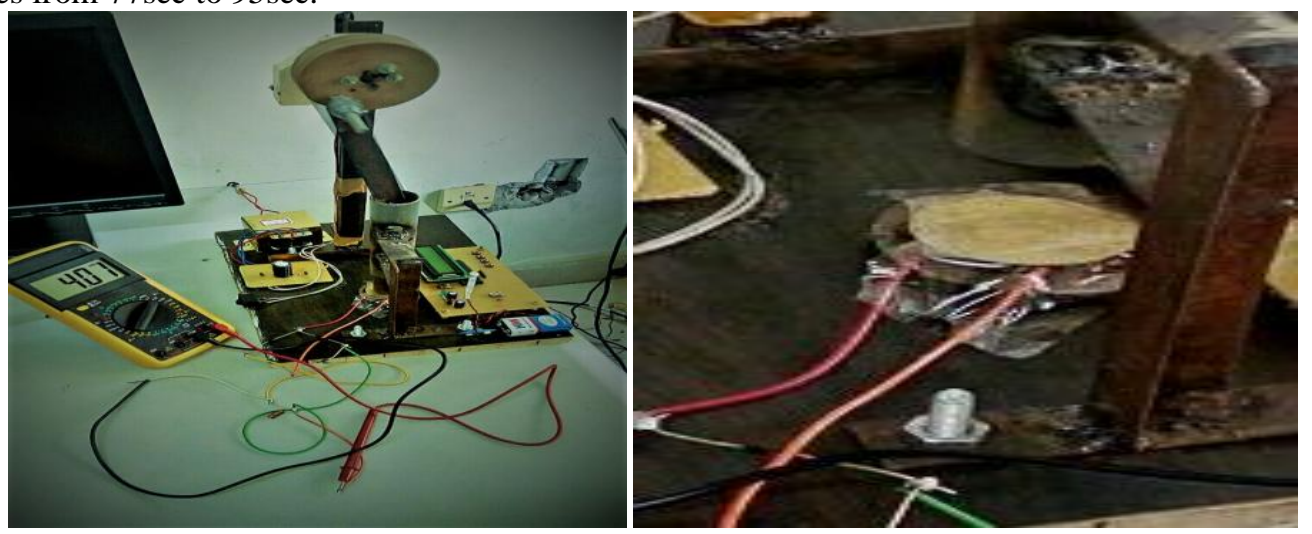

Fig: 10. Single Piezoelectric Full Wave Rectifier Circuit With Fixed Supported Boundary Condition

Table: 4. Boundary Condition: Fixed Supported

\begin{tabular}{|l|l|l|l|}
\hline Sr. No. & Output voltage & Load Reading & No. of Strokes \\
\hline 1. & 7.50 & 50 & 35 \\
\hline 2. & 4.25 & 55 & 40 \\
\hline 3. & 3.85 & 57 & 43 \\
\hline
\end{tabular}




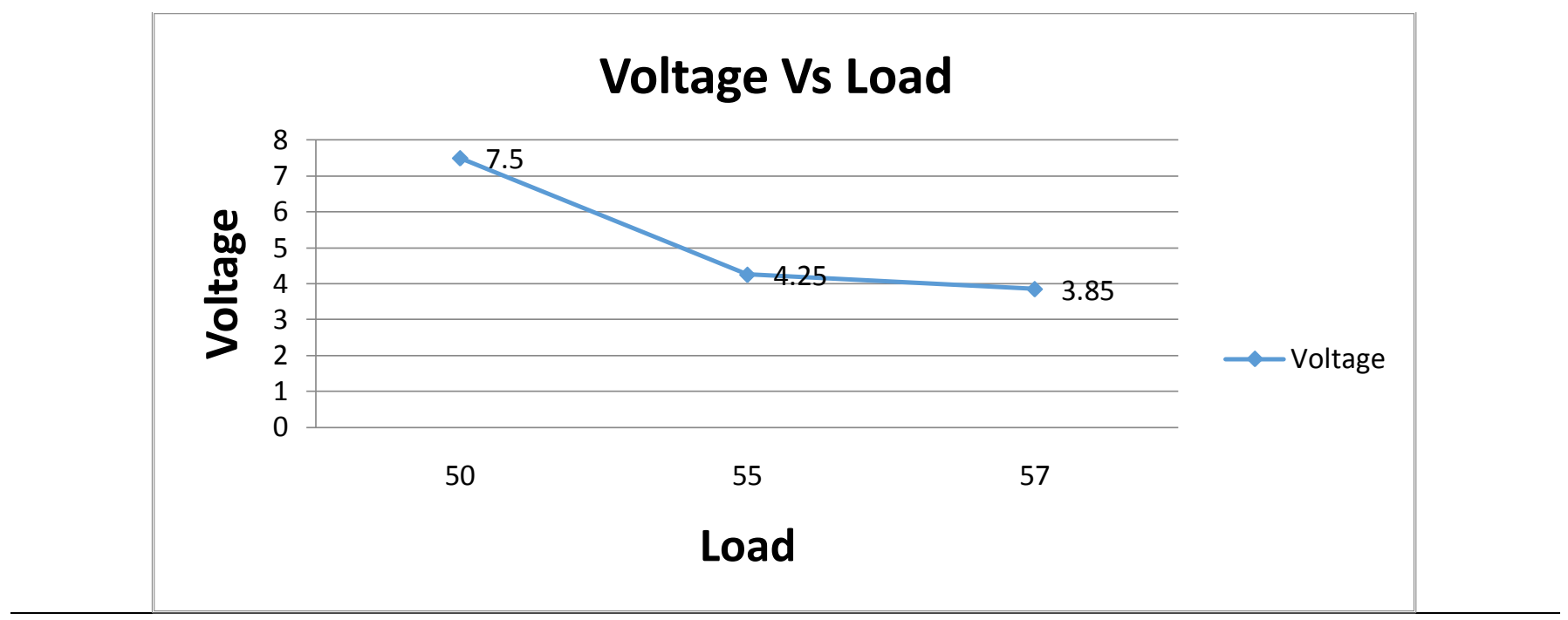

Fig: 11. Variation Of Voltage W.R.T. Load Applied For Fixed Supported Boundary Condition

\section{CONCLUSION:}

The work is done on designing a mechanism which apply periodic dynamic load on Piezo patch with different boundary conditions. The output voltage produced due to the application of periodic dynamic load is plotted versus applied load. It is noted that in each boundary condition after a small fluctuation in initial observations, the voltage output becomes almost steady with respect to applied load. It is also observed that in circular boundary condition, both output voltage as well as load value increase simultaneously. Simply supported takes much time (more strokes) than that of other boundary conditions. Comparatively, it is noted that for a load reading of 50, fixed support gives 7.5 volt output and simply support gives around 13 volt output. In constructing the mechanism to apply periodic dynamic load, several electronic parts are required like force sensor, printed circuit board and power source.

\section{REFRENCES:}

[1] Jonghyun Ryu Jaehoon Jung Seungmoon Choi, "Perceived Magnitudes of Vibrations Transmitted Through Mobile Device"

[2] Evgeniy L. Eremin, Evgeniy A. Shelenok “Automatic Control System of Testing Vibration Shaker"

[3] Waluyo Adi Siswanto, Mohd Norihan Ibrahim, Mohd Amran Madlan, and Siti Mariah Mohammad "Shaker Table Design for Electronic Device Vibration Test System"

[4] Deepak Chhabra and Ankit Singroha, "Analysis of Piezo polymer energy harvesting in different applications via diverse circuits"

[5] Nathaniel J. Guilar, Member, IEEE, Rajeevan Amirtharajah, Member, IEEE, and Paul J. Hurst, Fellow, IEEE "A Full-Wave Rectifier With Integrated Peak Selection for Multiple Electrode Piezoelectric Energy Harvesters"

[6] Deepak Chhabra and Ashwani Kumar 2016 "Study of PEH Configurations \& Circuitry and Techniques for Improving PEH Efficiency" International Journal for Scientific Research \& Development| Vol. 4, Issue 03, 2016

[7] Deepak Chhabra and Pooja Rani 2016 "Piezoelectric Energy Harvesting from Fluid Flow Dynamism using PVDF" International Journal of R\&D in Engineering, Science and Management Vol.4, Issue 1, May 2016, p.p.23-36, ISSN 2393-865X

[8] Deepak Chhabra and Anmol Budhwar 2016 "Comparison of Energy Harvesting using Single and Double Patch PVDF with Hydraulic Dynamism Anmol" International Journal of R\&D in Engineering, Science and Management Vol.4, Issue 1, May 2016, p.p.56-67, ISSN 2393-865X

[9] Xu Wang and Liwei Lin 2013 "Dimensionless optimization of piezoelectric vibration energy harvesters with different interface circuits" Smart Material Structure 22 (2013) 085011 (20pp)

[10] Chhabra, D., G. Bhushan and P. Chandna. 2013 "Optimal Placement of Piezoelectric Actuators on Plate Structures for Active Vibration Control Using Modified Control Matrix and Singular Value Decomposition Approach" International Journal of Mechanical Science and Engineering 7(3).

[11] Sood R, Jeon Y B,, Jeong H H and Kim S G 2005 “MEMS power generator with transverse mode thin film PZT” Sensors Actuators A 122 16-22 